\title{
miR-148 targets human DNMT3b protein coding region
}

\author{
ANJA M. DUURSMA, MARTIJN KEDDE, MARIETTE SCHRIER, CARLOS LE SAGE, and REUVEN AGAMI \\ Division of Tumor Biology, The Netherlands Cancer Institute, 1066 CX Amsterdam, The Netherlands
}

\begin{abstract}
MicroRNAs (miRNAs) are small noncoding RNA molecules of 20-24 nucleotides that regulate gene expression. In animals, miRNAs form imperfect interactions with sequences in the $3^{\prime}$ Untranslated region (3'UTR) of mRNAs, causing translational inhibition and mRNA decay. In contrast, plant miRNAs mostly associate with protein coding regions. Here we show that human miR-148 represses DNA methyltransferase $3 b(D n m t 3 b)$ gene expression through a region in its coding sequence. This region is evolutionary conserved and present in the Dnmt3b splice variants Dnmt3b1, Dnmt3b2, and Dnmt3b4, but not in the abundantly expressed Dnmt3b3. Whereas overexpression of miR-148 results in decreased DNMT3b1 expression, short-hairpin RNAmediated miR-148 repression leads to an increase in DNMT3b1 expression. Interestingly, mutating the putative miR-148 target site in Dnmt3b1 abolishes regulation by miR-148. Moreover, endogenous Dnmt3b3 mRNA, which lacks the putative miR-148 target site, is resistant to miR-148-mediated regulation. Thus, our results demonstrate that the coding sequence of Dnmt3b mediates regulation by the miR-148 family. More generally, we provide evidence that coding regions of human genes can be targeted by miRNAs, and that such a mechanism might play a role in determining the relative abundance of different splice variants.
\end{abstract}

Keywords: miRNA; coding region; DNMT3b; splice variants

\section{INTRODUCTION}

MiRNAs are small noncoding RNAs that have been identified as post-transciptional regulators of mRNA expression in many multicellular organisms such as plants, insects, and mammals. In animals, miRNAs have been shown to inhibit mRNA translation and to decrease mRNA stability by binding sequences in the 3'UTR (Wu et al. 2006; Standart and Jackson 2007). Perfect binding of nucelotides 2-7, the miRNA "seed," to the target mRNA is considered to play a key role in target recognition (Doench and Sharp 2004; Lewis et al. 2005). Binding of other miRNA nucleotides has been suggested to be involved in mRNA repression as well, but in this instance no perfect complementarity is required (Grimson et al. 2007).

In plants, most described miRNAs bind the protein coding sequence (CDS) of their target mRNAs with very highsequence complementarity, and this induces translational repression or RNA degradation in a way similar to RNA interference (Llave et al. 2002; Rhoades et al. 2002; Chen

Reprint requests to: Reuven Agami, Division of Tumor Biology, The Netherlands Cancer Institute, 1066 CX Amsterdam, The Netherlands; e-mail: r.agami@nki.nl; fax: 31-20-5122029.

Article published online ahead of print. Article and publication date are at http://www.rnajournal.org/cgi/doi/10.1261/rna.972008.
2004). The existence of miRNA-binding sites in animal CDS has been predicted by computational approaches. By analyzing mRNAs for conserved complementarity to the miRNA seed sequence, about a thousand target sites were predicted to occur in vertebrate CDSs (Lewis et al. 2005) and a similar approach in flies predicted sites in the CDSs as well (Stark et al. 2007). Furthermore, by combining computational approaches and human mRNA expression data, effective miRNA sites were detected in human CDS (Grimson et al. 2007). In an experimental assay, let-7-mediated repression was observed in zebrafish embryos when a let-7 target site was fused to the GFP CDS (Kloosterman et al. 2004). Furthermore, a miRNA pull-down of mRNAs and subsequent validation of predicted CDS sites of these mRNAs in reporter assays showed that fly miRNAs can target CDSs (Easow et al. 2007). Yet, to our knowledge, no functional miRNA-binding sites have been described in mammalian coding regions.

DNA methylation is an epigenetic modification that is involved in gene silencing, chromatin remodeling, and genome stability (Jones and Baylin 2007). In mammals, DNA methyltransferases attach methyl groups to cytosine residues of long stretches of CpG dinucleotides (CpG islands) that occur in repetitive sequences or in gene promoter regions (Gal-Yam et al. 2008). DNMT3a and DNMT3b have been identified as "de novo" methyltransferase, which 
methylate DNA during early development and gametogenesis (Okano et al. 1999). Inactivation of Dnmt3b results in embryonic lethality and Dnmt3a knock-out mice die shortly after birth (Okano et al. 1999). Although DNMT3a and DNMT3b have overlapping functions in imprinting of genes, DNMT3b was shown to be essential for methylation of centromeric minor satellite repeats (Okano et al. 1999). Furthermore, mutations in the human Dnmt3b gene have been linked to the ICF (Immunodeficiency, Centromere instability and Facial anomalies) syndrome (Xu et al. 1999). Lymphocytes from these patients display hypomethylation of centromeric repeat sequences, chromatin decondensation, and genomic instability (Jeanpierre et al. 1993; Tuck-Muller et al. 2000). In cancer, single nucleotide polymorphisms in the Dnmt3b promoter that were hypothesized to result in elevated Dnmt3b expression have been correlated with an increased risk of lung cancer (Shen et al. 2002; Lee et al. 2005). Interestingly, a recent study showed direct regulation of the Dnmt3a and Dnmt3b 3'UTRs by miR-29 and reduced expression of miR-29 correlated inversely with increased expression of these Dnmts in lung cancer tissue (Fabbri et al. 2007).

Dnmt3b has several splice variants, of which Dnmt3b1 and Dnmt3b3 are expressed most abundantly (Robertson et al. 1999). DNMT3b1 and DNMT3b2 have been shown to be catalytically active (Okano et al. 1998), whereas the role of DNMT3b3 in DNA methylation is not fully understood. Murine DNMT3b3 is catalytically inactive both in vitro (Aoki et al. 2001) and in vivo (Chen et al. 2003); however, the DNA methyltransferase activity of human DNMT3b3 depended on which substrate was chosen (Soejima et al. 2003; Chen et al. 2005). This discrepancy might reflect a change in target preference since Dnmt3b3, but also Dnmt3b4, lack a motif that could be responsible for target recognition (Kumar et al. 1994). Interestingly, high expression of splice variant Dnmt3b4 has been associated with DNA hypomethylation at pericentromeric satellite regions (Saito et al. 2002). This could be a result of dominantnegative regulation or an example of changed target preference. The latter could result in altered gene expression, and this was indeed observed by microarray analysis in cells that exogenously expressed Dnmt3b7, a splice variant that was found highly expressed in cancer cells (Ostler et al. 2007). Another possibility would be that DNMT3b3 stimulates DNMT3b1 catalytic activity in a similar way to the DNMT3b-related protein DNMT3L, which also lacks methyltransferase activity (Chen et al. 2005).

Here, we describe our finding that human miR-148 regulates DNMT3b expression through interaction with a conserved site in its protein coding sequence. Interestingly, the target site is absent in the abundantly expressed Dnmt3b3 splice variant, making it resistant to miRNA-mediated regulation. Thus, the expression of miR148 changes the relative abundance of DNMT3b splice variants.

\section{RESULTS AND DISCUSSION}

In plants, most miRNAs regulate mRNA expression by interacting with highly homologous CDS (Rhoades et al. 2002). We identified a site in the Dnmt3b CDS (nucleotides 2384-2411) that is highly homologous to the miR-148 family (Fig. 1A). This region has the potential to interact with all 22 nucleotides (nt) of miR-148a and miR-148b (leaving only one gap) and therefore resembles miRNA target interactions observed in plants. Importantly, the target site (referred to as site \#1) is conserved in rhesus, mouse, rat, dog, horse, and armadillo Dnmt3b1, Dnmt3b2, and Dnmt3b4, suggesting that this sequence is retained through evolution (Fig. 1A). However, this particular sequence is not present in the Dnmt3b3 splice variant (Fig. $1 \mathrm{~B}$; data not shown). Another site (\#2) that contains complementarity to the miR-148 family is situated between nucleotides 1424 and 1439 (determined using targetscanS and RNA22) (Fig. 1B). Although it has a good match of the miRNA seed sequence (nucleotides 2-7), which is thought to specify miRNA targets in $3^{\prime}$ UTRs (Doench and Sharp 2004; Lewis et al. 2005), extensive complementarity such as observed with site \#1 is not seen.

Next, we designed experiments to study the regulation of Dnmt3b by miR-148. First, we used miR-Vec-148a, a miRNA-expression vector for ectopic expression of miR148a (Voorhoeve et al. 2006). Using an RNase protection
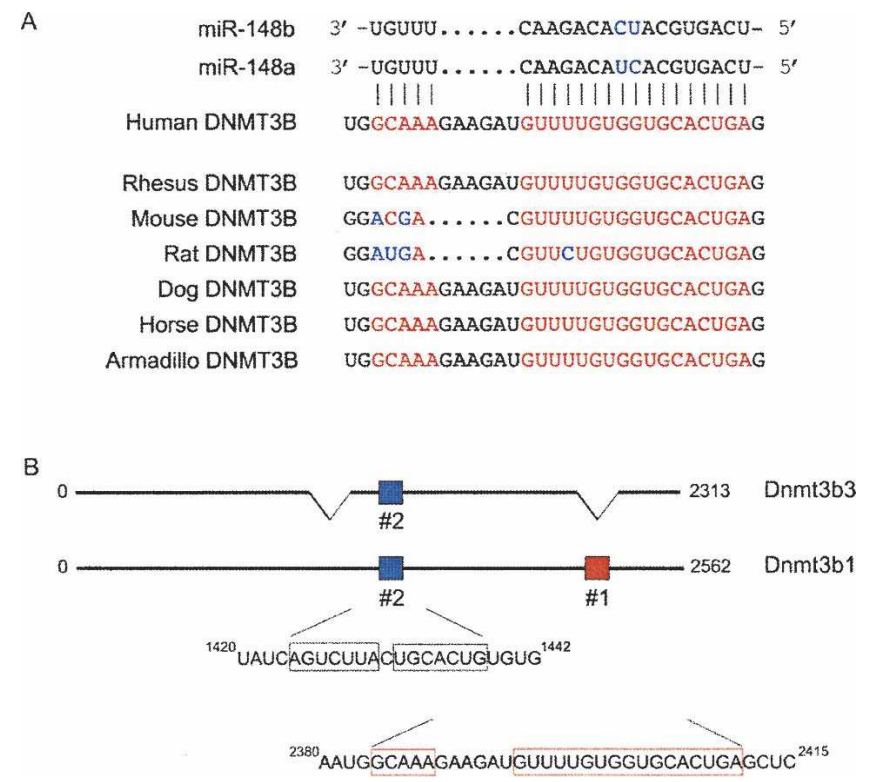

FIGURE 1. Schematic representation of putative miR-148 sites in the Dnmt3b1 coding region. (A) miR-148a and miR-148b are highly complementary to nucleotides $2382-2412$ of human Dnmt3b. This potential miR-148 target site is conserved in rhesus, mouse, rat, dog, horse, and armadillo. This site is referred to as target site \#1. (B) Schematic representation of two possible miR-148 target sites in the Dnmt3b coding region. 
assay (RPA) for miR-148 in HeLa cells, which were previously shown to be negative for miR-148 (Barad et al. 2004), transient and stable introduction of miR-Vec-148a led to high expression of this miRNA (Fig. 2A). Second, we determined whether we could identify cell lines that endogenously express miR-148 by quantitative real-time (qRT) PCR specific for mature miR-148a. We observed high miR-148 expression in the 2102EP testicular germ-cell line, 293T cells, Jurkat T cells, but not in HeLa, MCF-7 cells, and diploid BJ fibroblasts that were immortalized with telomerase (Fig. 2B). Notably, the high expression observed in the transient transfected miR-148a HeLa cells was very similar to the endogenous expression of miR-148a found in Jurkat cells (Fig. 2B). In contrast to miR-148a, we were unable to detect endogenous expression miR-148b (which was originally cloned from rat) in human cell lines (data not shown). Third, we cotransfected miR-Vec-148a with an expression vector containing the coding region for Dnmt3b1 downstream of the green fluorescent protein (GFP-Dnmt3b1) and performed immunoblotting analysis for GFP. Notably, Dnmt3b was cloned in-frame with GFP, which was verified by sequencing. Furthermore, the translational fusion product was detected at the appropriate size

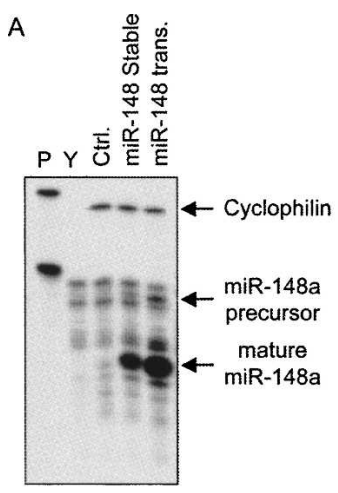

B

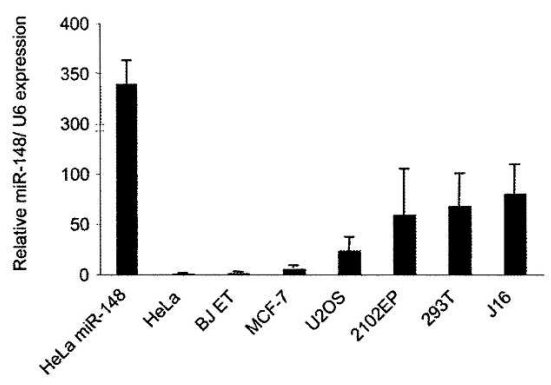

C
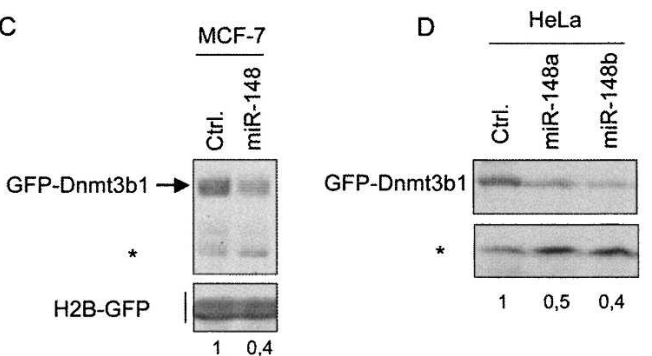

$E$

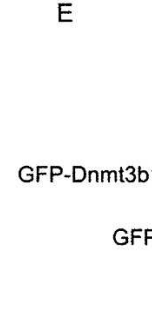

by Immunoblotting analysis (data not shown). We observed a marked reduction of $60 \%$ of GFP-DNMT3b1 expression, but not of cotransfected H2B-GFP, in cells expressing miR-148 (Fig. 2C). As expected from the sequence similarity between miR-148a and miR-148b, a similar reduction of GFP-DNMT3b1 expression in cells expressing miR-148b was observed (Fig. 2D). These results suggest that the presence of miR-148 represses the expression of DNMT3b1.

To determine whether the observed reduction in DNMT3b1 expression by miR-148 is dependent on the putative miR-148 target sites, we mutated these regions to significantly reduce recognition by the miRNAs. Importantly, mutating these sites did not change the frame of the fusion proteins, as was verified by sequencing and immunoblotting analysis (data not shown). Transient cotransfection experiments revealed that mutating the highly homologous target site \#1 almost completely abrogated miR-148-mediated regulation (Fig. 2E). However, a mutant of target site \#2, which has much less complementarity to miR-148, still retained sensitivity to miR-148. This suggests that miR-148 targets Dnmt3b1 through a highly complementary sequence in the Dnmt3b1 coding region.

The results above imply that the Dnmt3b3 splice variant, which lacks miR-148 target site \#1, is resistant to miR-148-mediated regulation. To examine this issue, we designed primers for qRT-PCR that distinguish endogenous Dnmt3b3 from Dnmt3b1 and other variants that express the targeted exon such as Dnmt3b2 and Dnmt3b4 mRNA (Fig. 3A). This analysis revealed that stable expression of miR-148a in HeLa cells resulted in small but significant reduction $(P<0.001)$ in Dnmt3b1 mRNA levels of $25 \%$, while no reduction in Dnmt3b3 mRNA levels was observed (Fig. 3A). Thus, the expression of miR$148 \mathrm{a}$ affects the mRNA stability of Dnmt3b1, but not Dnmt3b3, leading to a relative increase in the abundance of Dnmt3b3 compared with Dnmt3b1 and all other Dnmt3b splice variants that express the targeted site.

DNMT3b protein is highly expressed in undifferentiated embryonic stem cells, whereas its expression level is much reduced in somatic cells (Okano et al. 1998). To study the effect of miR$148 \mathrm{a}$ on endogenous DNMT3b protein expression, we attempted to detect it in several tumor cell lines using immunoblot analysis. In our hands, only 2102EP testicular germ-cell tumor cells 
A
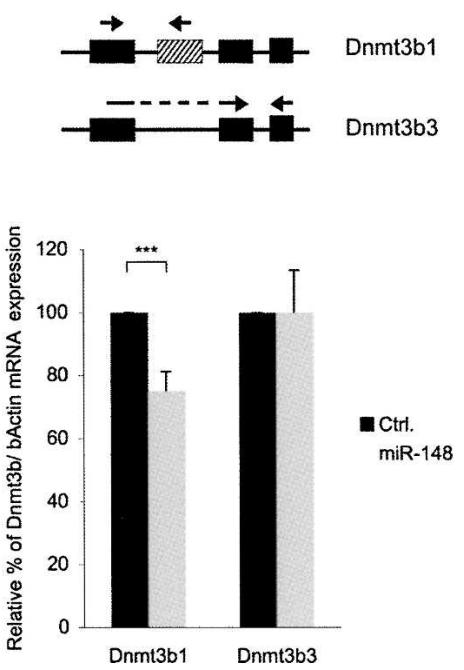

FIGURE 3. miR-148 reduces endogenous Dnmt3b1 mRNA and protein level. (A) qRT-PCR of either miR-Vec-148 or control (Ctrl.) transfected HeLa cells. Specific primers were used to detect Dnmt3b1 and Dnmt3b3 as shown schematically. Error bars represent standard deviation, $n=4$ and $\left(^{* * *}\right) P<0.001$. (B) $2102 \mathrm{EP}$ cells were electroporated with the indicated constructs and subjected to immunoblot analysis for DNMT3b and TUBULIN.

expressed endogenous DNMT3b in sufficient amounts to allow detection by Western blot (data not shown). Furthermore, these cells express moderate levels of miR-148a (Fig. 2B). To assess whether increasing the miR-148 level would result in a reduced endogenous DNMT3b1 protein level, we electroporated the miR-148 expression vector into 2102EP cells and subjected them to immunoblotting analysis with a specific anti-Dnmt3b antibody. In line with the results above, overexpression of miR-148a reduced DNMT3b1 protein levels up to $70 \%$ compared with two different miRNA control constructs (Fig. 3B). We used two short-hairpin RNA (shRNA) constructs targeting Dnmt3b and an expression vector for Dnmt3b1 to control for the specificity of the Dnmt3b antibody (Fig. 3B).

Finally, we asked whether inhibition of miR-148a activity would elevate DNMT3b1 expression. We designed two shRNA constructs targeting the miR-148 precursor RNA. Electroporation of $21202 \mathrm{EP}$ cells with these constructs showed that one construct, miR- $148^{\mathrm{kd} \# 2}$, was functional in reducing endogenous miR-148 levels as determined by RPA analysis (Fig. 4A). Western blot analysis of cells electroporated with miR-148 ${ }^{\mathrm{kd} \# 2}$ indeed revealed an increase in the DNMT3b1 protein levels (Fig. 4B). We therefore conclude that endogenous DNMT3b1 expression is controlled by miR-148a in $2102 \mathrm{EP}$ cells.

Our data reveal a novel function of miRNA-mediated regulation. miRNA targeting of human coding regions in addition to $3^{\prime}$ UTRs not only extends the range of potential target sites, but also allows for splice variant-specific regulation. Significantly, different DNMT3b splice variants might play a role in regulating DNMT3b activity or target-site preference. This could be an additional mechanism to the regulation of splice variant abundance by alternative splicing. Our results rule out the possibility that alternative splicing is induced by miR-148 targeting, since miR-148 reduced expression of exogenous DNMT3b-GFP in a site-specific manner.

It is interesting that only the high-affinity miR-148-target site in Dnmt3b1 was affected by miR-148. In the future, it will be important to determine whether miRNA binding to CDS requires more complementarity with the target than interactions with $3^{\prime}$ UTRs. Plant miRNAs interact with high-sequence complementarity with their target CDS eliciting, in most of the cases, an RNA interference-mediated cleavage of the target mRNA (Llave et al. 2002; Rhoades et al. 2002). Although we observed reduction of Dnmt3b mRNA in the presence of miR-148, the reduction was less prominent than the observed reduction on protein levels. This suggests that miR-148 induces both translational repression and mRNA degradation of Dnmt3b1. Further experiments should approach this point.

The mechanism by which miRNAs mediate repression is not completely understood. Whereas some evidence exists for interference with initiation of translation, others suggest that miRNAs prevent the "closed loop" mRNA configuration induced by interaction of poly(A)-binding proteins
A

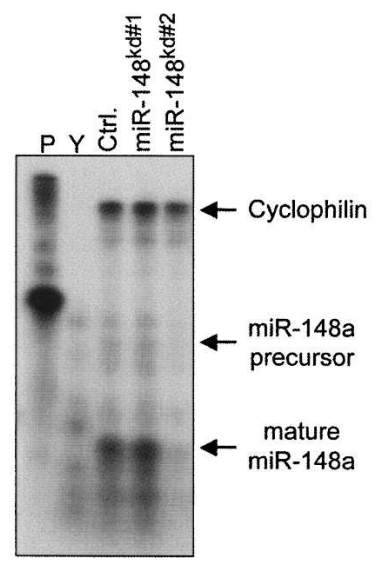

B

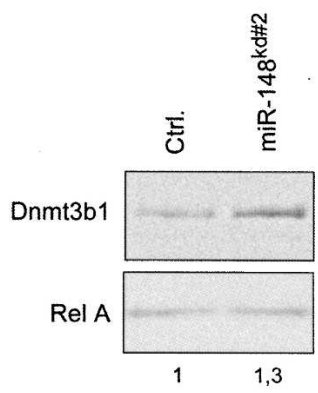

FIGURE 4. Suppression of endogenous miR-148 increases DNMT3b1 expression. (A) miR-148a level was detected by RPA as described in Figure 2A. (B) 2102EP cells were electroporated with either control (Ctrl.) or miR-148 ${ }^{\mathrm{kd} \# 2}$ constructs and subjected to immunoblot analysis with Dnmt3b and Rel A antibodies. 
with initiation factors at the 5' cap (Standart and Jackson 2007). The latter enhances translation efficiency. miR-148 targets Dnmt3b1 $\sim 150$ nt before the stop codon in the CDS, which is near the $3^{\prime} \mathrm{UTR}$ and the poly(A) tail. Therefore, the location of the miR-148 target site might allow for miRNA-mediated repression in a similar fashion as $3^{\prime}$ UTR binding miRNAs. On the other hand, experiments with miRNA target sites cloned in the $5^{\prime}$ UTR of a luciferase construct showed miRNA-mediated repression (Lytle et al. 2007), suggesting that repression might be independent of the target site location. The exact mechanism through which miR-148 targets Dnmt3b1 remains to be explored.

In conclusion, we present evidence for a functional interaction of human miR-148 with the Dnmt3b1 CDS. It would be interesting to see whether miRNA-mediated regulation of protein coding regions turn out to be a common theme in miRNA targeting of mammalian mRNAs or the exception that confirms the rule.

\section{MATERIALS AND METHODS}

\section{Constructs and antibodies}

Dnmt3b expression constructs were cloned by PCR in pEGFP-C2 (Clontech). Dnmt3b mutant constucts were generated by sitedirected mutagenesis using PCR, and constructs were verified by DNA sequence analysis. Mutant\#1 ${ }^{1429}$ TACTGCACTG ${ }^{1438}$ was replaced by TTCTCGTCA. Mutant\#2 ${ }^{2398}$ TTGTGGTGCACT ${ }^{2409}$ was replaced by TTAGCCGCGACC. All miRNAs were expressed from a retroviral miR-Vec vector as described before (Voorhoeve et al. 2006). We used pSuper short-hairpin constructs (Brummelkamp et al. 2002) targeting Dnmt3b and miR-148: Dnmt3b ${ }^{\text {kd }}$ \#1 AGAT GACGGATGCCTAGAG Dnmt3b $\mathrm{b}^{\mathrm{kd}} \# 2$ (AGGTAGGAAAGTACGT CGC) $\mathrm{miR}-148^{\mathrm{kd}}$ \#1 ACTCTGAGTATGATAGAAG miR-148 $8^{\mathrm{kd}}$ 2 GTCAGTGCACTACAGAACT. Antibodies against Dnmt3b (H-230, Santa Cruz), Tubulin (YL1/2, ECACC), and NFkB p65 (C-20 Santa Cruz) and GFP (a gift from J. Neefjes) were used.

\section{Cell culture and transfection}

Hela, 2102EP, 293T, MCF-7, U2OS, BJ ET primary fibroblasts (expressing the ecotrophic receptor and human telomerase) were grown in Dulbecco's Modified Eagle Medium and Jurkat cells in Iscove's Modified Dulbecco's Medium suplemented with $10 \%$ fetal bovine serum. Hela cells were transfected with Fugene (Roche) using the manufacturer's protocol. $2102 \mathrm{EP}$ cells were transfected by electroporation as described (Agami and Bernards 2000). A Gene Pulser Xcell Electroporation System (Bio-Rad) was used at 120 volts, $10 \times 1.5 \mathrm{msec}$ burst duration and $1 \mathrm{sec}$ interval.

\section{Immunoblotting}

For Western blot analysis, whole-cell extracts were separated at $6 \%$ SDS-PAGE minigels and transferred to polyvinylidene difluoride membranes (Millipore). Western blots were developed using enhanced chemiluminescence (Amersham Biosciences). Densito- metric quantification of Western blot assays was done by using Tina (version 2.09f) software.

\section{RNA isolation, RPA, and real-time RT-PCR}

RNA was isolated from cells using Trizol (Invitrogen). RNase protection assays (RPAs) were performed using the mirVana miRNA probe construction and detection kits (Ambion) according to the manufacturers' protocol. A total of $5 \mu \mathrm{g}$ of RNA was used per assay and a ATAGAAGTCAGTGCACTACAGAACTTTG TCTCCCTGTCTC primer was used to make a miR-148a probe. Quantification was performed by densitometry.

cDNA was prepared from $1.5 \mu \mathrm{g}$ of RNA using random hexamer primers (Superscript III first-strand synthesis system for RT-PCR, Invitrogen). Quantitative RT-PCR was performed with a standard two-step amplification protocol of a MiniOpticon System (Bio-Rad) apparatus using a SYBRgreen PCR master mix (Applied Biosystem) and specific primers:

\section{Dnmt3b1 forward GCCGTTCTTCTGGATGTTTGAG; Dnmt3b1 reverse ATCCTATTGTATTCCAAGCAGTCC; Dnmt3b3 forward ATCTCACGGTTCCTGGAGTG; Dnmt3b3 reverse AAGCCAAAGATCCTGTTCATCC; $\beta$ Actin forward CCTGGCACCCAGCACAAT; and $\beta$ Actin reverse GGGCCGGACTCGTCATACT.}

The mirVana qRT-PCR miRNA detection kit (Ambion) was used to detect miRNA expression by quantitative RT-PCR. Specific miR-148a and U6 RT and PCR primers were used (Ambion) and $25 \mathrm{ng}$ of RNA was used per reaction.

\section{ACKNOWLEDGMENTS}

We thank all members of the Agami laboratory for stimulating discussions. This work was supported by the Dutch Cancer Society (KWF) and the European Young Investigator award (EURYI) to R.A.

Received December 21, 2007; accepted February 6, 2008.

\section{REFERENCES}

Agami, R. and Bernards, R. 2000. Distinct initiation and maintenance mechanisms cooperate to induce G1 cell cycle arrest in response to DNA damage. Cell 102: 55-66.

Aoki, A., Suetake, I., Miyagawa, J., Fujio, T., Chijiwa, T., Sasaki, H., and Tajima, S. 2001. Enzymatic properties of de novo-type mouse DNA (cytosine-5) methyltransferases. Nucleic Acids Res. 29: 35063512. doi: 10.1093/nar/29.17.3506.

Barad, O., Meiri, E., Avniel, A., Aharonov, R., Barzilai, A., Bentwich, I., Einav, U., Gilad, S., Hurban, P., Karov, Y., et al. 2004. MicroRNA expression detected by oligonucleotide microarrays: System establishment and expression profiling in human tissues. Genome Res. 14: 2486-2494.

Brummelkamp, T.R., Bernards, R., and Agami, R. 2002. A system for stable expression of short interfering RNAs in mammalian cells. Science 296: 550-553.

Chen, X. 2004. A microRNA as a translational repressor of APETALA2 in Arabidopsis flower development. Science 303: 20222025.

Chen, T., Ueda, Y., Dodge, J.E., Wang, Z., and Li, E. 2003. Establishment and maintenance of genomic methylation patterns 
in mouse embryonic stem cells by Dnmt3a and Dnmt3b. Mol. Cell. Biol. 23: 5594-5605.

Chen, Z.X., Mann, J.R., Hsieh, C.L., Riggs, A.D., and Chedin, F. 2005. Physical and functional interactions between the human DNMT3L protein and members of the de novo methyltransferase family. J. Cell. Biochem. 95: 902-917.

Doench, J.G. and Sharp, P.A. 2004. Specificity of microRNA target selection in translational repression. Genes \& Dev. 18: 504-511.

Easow, G., Teleman, A.A., and Cohen, S.M. 2007. Isolation of microRNA targets by miRNP immunopurification. RNA 13: 1198-1204.

Fabbri, M., Garzon, R., Cimmino, A., Liu, Z., Zanesi, N., Callegari, E., Liu, S., Alder, H., Costinean, S., Fernandez-Cymering, C., et al. 2007. MicroRNA-29 family reverts aberrant methylation in lung cancer by targeting DNA methyltransferases $3 \mathrm{~A}$ and 3B. Proc. Natl. Acad. Sci. 104: 15805-15810.

Gal-Yam, E.N., Saito, Y., Egger, G., and Jones, P.A. 2008. Cancer epigenetics: Modifications, screening, and therapy. Annu. Rev. Med. 59: 267-280.

Grimson, A., Farh, K.K., Johnston, W.K., Garrett-Engele, P., Lim, L.P., and Bartel, D.P. 2007. MicroRNA targeting specificity in mammals: Determinants beyond seed pairing. Mol. Cell 27: 91-105.

Jeanpierre, M., Turleau, C., Aurias, A., Prieur, M., Ledeist, F., Fischer, A., and Viegas-Pequignot, E. 1993. An embryonic-like methylation pattern of classical satellite DNA is observed in ICF syndrome. Hum. Mol. Genet. 2: 731-735.

Jones, P.A. and Baylin, S.B. 2007. The epigenomics of cancer. Cell 128: 683-692.

Kloosterman, W.P., Wienholds, E., Ketting, R.F., and Plasterk, R.H. 2004. Substrate requirements for let-7 function in the developing zebrafish embryo. Nucleic Acids Res. 32: 6284-6291.

Kumar, S., Cheng, X., Klimasauskas, S., Mi, S., Posfai, J., Roberts, R.J., and Wilson, G.G. 1994. The DNA (cytosine-5) methyltransferases. Nucleic Acids Res. 22: 1-10. doi: 10.1093/nar/22.1.1.

Lee, S.J., Jeon, H.S., Jang, J.S., Park, S.H., Lee, G.Y., Lee, B.H., Kim, C.H., Kang, Y.M., Lee, W.K., Kam, S., et al. 2005. DNMT3B polymorphisms and risk of primary lung cancer. Carcinogenesis 26: 403-409.

Lewis, B.P., Burge, C.B., and Bartel, D.P. 2005. Conserved seed pairing, often flanked by adenosines, indicates that thousands of human genes are microRNA targets. Cell 120: 15-20.

Llave, C., Xie, Z., Kasschau, K.D., and Carrington, J.C. 2002. Cleavage of Scarecrow-like mRNA targets directed by a class of Arabidopsis miRNA. Science 297: 2053-2056.

Lytle, J.R., Yario, T.A., and Steitz, J.A. 2007. Target mRNAs are repressed as efficiently by microRNA-binding sites in the $5^{\prime}$ UTR as in the 3' UTR. Proc. Natl. Acad. Sci. 104: 9667-9672.

Okano, M., Xie, S., and Li, E. 1998. Cloning and characterization of a family of novel mammalian DNA (cytosine-5) methyltransferases. Nat. Genet. 19: 219-220.
Okano, M., Bell, D.W., Haber, D.A., and Li, E. 1999. DNA methyltransferases Dnmt3a and Dnmt3b are essential for de novo methylation and mammalian development. Cell 99: 247-257.

Ostler, K.R., Davis, E.M., Payne, S.L., Gosalia, B.B., ExpositoCespedes, J., Le Beau, M.M., and Godley, L.A. 2007. Cancer cells express aberrant DNMT3B transcripts encoding truncated proteins. Oncogene 26: 5553-5563.

Rhoades, M.W., Reinhart, B.J., Lim, L.P., Burge, C.B., Bartel, B., and Bartel, D.P. 2002. Prediction of plant microRNA targets. Cell 110: 513-520.

Robertson, K.D., Uzvolgyi, E., Liang, G., Talmadge, C., Sumegi, J., Gonzales, F.A., and Jones, P.A. 1999. The human DNA methyltransferases (DNMTs) 1, 3a and 3b: Coordinate mRNA expression in normal tissues and overexpression in tumors. Nucleic Acids Res. 27: 2291-2298. doi: 10.1093/nar/27.11.2291.

Saito, Y., Kanai, Y., Sakamoto, M., Saito, H., Ishii, H., and Hirohashi, S. 2002. Overexpression of a splice variant of DNA methyltransferase 3b, DNMT3b4, associated with DNA hypomethylation on pericentromeric satellite regions during human hepatocarcinogenesis. Proc. Natl. Acad. Sci. 99: 10060-10065.

Shen, H., Wang, L., Spitz, M.R., Hong, W.K., Mao, L., and Wei, Q. 2002. A novel polymorphism in human cytosine DNA-methyltransferase-3B promoter is associated with an increased risk of lung cancer. Cancer Res. 62: 4992-4995.

Soejima, K., Fang, W., and Rollins, B.J. 2003. DNA methyltransferase $3 \mathrm{~b}$ contributes to oncogenic transformation induced by SV40T antigen and activated Ras. Oncogene 22: 4723-4733.

Standart, N. and Jackson, R.J. 2007. MicroRNAs repress translation of m7Gppp-capped target mRNAs in vitro by inhibiting initiation and promoting deadenylation. Genes \& Dev. 21: 1975-1982.

Stark, A., Lin, M.F., Kheradpour, P., Pedersen, J.S., Parts, L., Carlson, J.W., Crosby, M.A., Rasmussen, M.D., Roy, S., Deoras, A.N., et al. 2007. Discovery of functional elements in 12 Drosophila genomes using evolutionary signatures. Nature 450: 219-232.

Tuck-Muller, C.M., Narayan, A., Tsien, F., Smeets, D.F., Sawyer, J., Fiala, E.S., Sohn, O.S., and Ehrlich, M. 2000. DNA hypomethylation and unusual chromosome instability in cell lines from ICF syndrome patients. Cytogenet. Cell Genet. 89: 121-128.

Voorhoeve, P.M., le Sage, C., Schrier, M., Gillis, A.J., Stoop, H., Nagel, R., Liu, Y.P., van Duijse, J., Drost, J., Griekspoor, A., et al. 2006. A genetic screen implicates miRNA-372 and miRNA-373 as oncogenes in testicular germ cell tumors. Cell 124: 1169-1181.

Wu, L., Fan, J., and Belasco, J.G. 2006. MicroRNAs direct rapid deadenylation of mRNA. Proc. Natl. Acad. Sci. 103: 4034-4039.

Xu, G.L., Bestor, T.H., Bourc'his, D., Hsieh, C.L., Tommerup, N., Bugge, M., Hulten, M., Qu, X., Russo, J.J., and ViegasPequignot, E. 1999. Chromosome instability and immunodeficiency syndrome caused by mutations in a DNA methyltransferase gene. Nature 402: 187-191. 

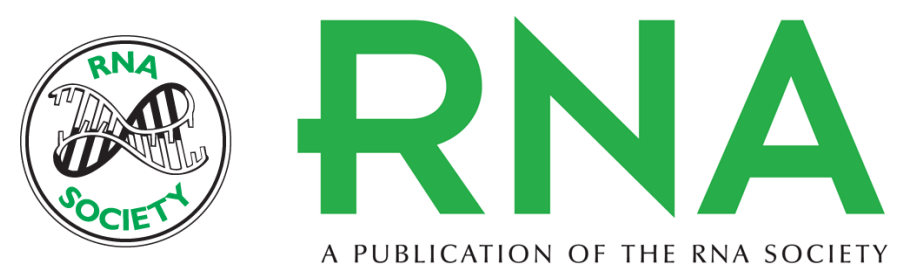

A PUBLICATION OF THE RNA SOCIETY

\section{miR-148 targets human DNMT3b protein coding region}

Anja M. Duursma, Martijn Kedde, Mariette Schrier, et al.

RNA 2008 14: 872-877

References This article cites 34 articles, 13 of which can be accessed free at:

http://rnajournal.cshlp.org/content/14/5/872.full.html\#ref-list-1

License

Email Alerting Receive free email alerts when new articles cite this article - sign up in the box at the Service top right corner of the article or click here. 